\title{
Special issue on graphene
}

Graphene, a two-dimensional sheet of $\mathrm{sp}^{2}$-hybridized carbon atoms, is considered to be the basic building block of other graphitic allotropes including three-dimensional graphite, one-dimensional carbon nanotubes and zero-dimensional fullerenes. Since its discovery by Geim and Novoselov in 2004, graphene has attracted considerable interest from researchers in materials science, condensed-matter physics, information science and chemistry. Graphene possesses a series of unique and fascinating properties such as quantum Hall effect, tunable band gap, extremely high mobility, remarkably high Young's modulus and very large specific surface area. These properties make graphene suitable for a wide variety of potential applications including transistors, transparent touch screens, light panels, and solar cells, as well as being a robust composite material for use in satellites, airplanes, and cars. Given the sheer scale of the potential impact of graphene, it is perhaps not surprising that Geim and Novoselov were awarded the Nobel Prize in Physics in 2010 because of their decisive contributions to graphene development. Taken together, it is envisaged that graphene will exert a far-reaching influence on science, technology and society.

Currently, there are several intensive research programs being conducted worldwide exploring (1) the electronic, optical, thermal, magnetic, structural and chemical properties of graphene; (2) the large-scale and controllable synthesis of graphene; (3) the assembly of graphene-based composites into well-controlled architectures; (4) novel techniques for the characterization of graphene; and (5) the fabrication, integration and applications of graphene devices, sensors and actuators. For those like myself, working in the field of photoelectronics, the achievements made in synthesizing large areas of high-quality graphene, and their subsequent application in transparent conductive films and electronic circuits, represent some of the most outstanding areas of progress that have emerged. Although realistic applications may be some years away, these developments have enabled the manufacture of flexible organic light emitting diode displays and all-carbon photoelectronic devices.

Chinese scientists have made many internationally welcomed contributions to graphene research. To highlight our current state and elevate our competitive capability at the very forefront of this rapidly evolving area of science, we have asked a number of domestic scientific groups in this field to provide papers on graphene for this special issue.

This special issue comprises 7 Reviews and 12 Articles. The reviews cover recent areas of progress in graphene research, including graphene synthesis and its properties and applications. A dozen original works on the preparation, modification, theoretical calculation and usage of graphene-based materials have been reported in the Articles. This issue highlights only a section of the work conducted by scientific groups based in China and it undoubtedly the case that many promising areas of progress have not been included. In spite of this, I believe that the research included in this issue will bring valuable information to the readers and the wider scientific community.

I would like to thank all of the authors for their contributions to this special issue. I would also like to thank the Chinese Science Bulletin for publishing this special issue and Ms. FU Li and Dr. ZHI Xin for their kind assistance and editing work. I would like to express my appreciation to the Editor-in-Chief and Academician of Chinese Academy of Sciences, Professor XIA Jianbai, for encouraging me to organize this special issue.

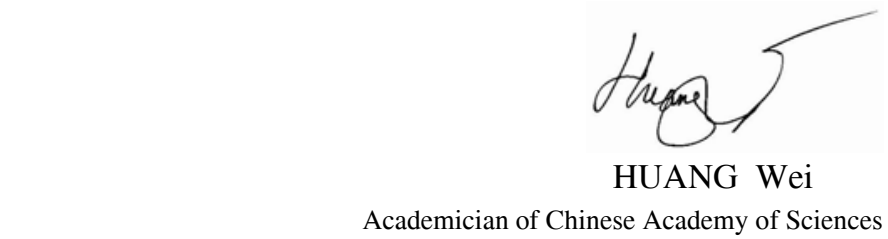

Nanjing University of Posts and Telecommunications, Nanjing 210046, China

Open Access This article is distributed under the terms of the Creative Commons Attribution License which permits any use, distribution, and reproduction in any medium, provided the original author(s) and source are credited. 